in vivo $35: 1117-1123(2021)$

doi:10.21873/invivo.12357

\title{
Outcome After Radiation Therapy in Canine Intracranial Meningiomas or Gliomas
}

\author{
TOMÁS RODRIGUES MAGALHÃES ${ }^{1}$, JÉRÔME BENOÎT ${ }^{2}$, SLAVOMÍRA NÉČOVÁ ${ }^{2}$, \\ SUSAN NORTH ${ }^{2}$ and FELISBINA LUÍSA QUEIROGA ${ }^{1,3,4}$ \\ ${ }^{1}$ Department of Veterinary Sciences, University of Trás-os-Montes and Alto Douro, Vila Real, Portugal; \\ ${ }^{2}$ Southfields Veterinary Specialists (formerly VRCC Veterinary Referrals), Essex, U.K.; \\ ${ }^{3}$ Center for Research and Technology of Agro-Environment and Biological Sciences (CITAB), \\ University of Trás-os-Montes and Alto Douro, Vila Real, Portugal; \\ ${ }^{4}$ Center for the Study of Animal Sciences, CECA-ICETA, University of Porto, Porto, Portugal
}

\begin{abstract}
Aim: To characterize a group of dogs diagnosed with meningioma or glioma treated with radiation therapy and assess the clinical impact of diagnosis and radiation protocol on survival time. Patients and Methods: Canine patient records from a single veterinary referral hospital, between 2011 and 2015, were searched for intracranial tumour cases treated with radiation therapy, as a sole modality. Thirty-two dogs were included. Results: Median survival times were 524 days [95\% confidence interval $(C I)=287-677]$ in total, 512 days (95\% CI=101-682) for the glioma group and 536 days (95\% CI=249-677) for the meningioma group. No significant difference in survival was detected when using a definitive or a palliative protocol $(p=0.130)$, nor other prognostic factors were found. Conclusion: Our results highlight the efficacy of radiation therapy in the treatment of canine meningioma, as well as glioma, suggesting a change in the current perception of the response of glial tumours to radiation.
\end{abstract}

The two main primary brain tumours described in dogs are meningiomas and gliomas, with an approximate representation of $50 \%$ and $35 \%$, respectively. Meningiomas develop outside the brain parenchyma (extra-axial) and are associated, in most of its variants, with a benign behavior, yet more aggressive anaplastic meningiomas are also

This article is freely accessible online.

Correspondence to: Felisbina Queiroga, Department of Veterinary Sciences, University of Trás-os-Montes and Alto Douro, Quinta dos Prados, 5000-801 Vila Real, Portugal. Tel: +351 917826982, Fax: +351 259350480, e-mail: fqueirog@utad.pt

Key Words: Brain, dogs, glioma, intracranial, meningioma, tumours, radiation. described. Glial tumours originate from the brain parenchyma (intra-axial) and are differentiated in three main categories: oligodendroglioma, astrocytoma and undefined glioma. The associated clinical signs are the result of its continuous growth and expansion, which lead to increased pressure in the adjacent brain parenchyma, and also due to secondary events, such as inflammation, edema, ischemia, hemorrhage and, in some cases, obstructive hydrocephalus and brain herniation (1).

Although definitive diagnosis is only possible by histopathological analysis of the tumour, the difficulty in performing this exam ante-mortem, leads to the fact that most neoplasms are diagnosed, in the literature, through clinical presentation and imaging features (2-7). Thus, computed tomography $(\mathrm{CT})$ and magnetic resonance imaging (MRI) are two imaging techniques widely used to establish a presumptive diagnosis of a brain tumour. MRI is considered a diagnostic method with greater accuracy for brain tumours and enables the evaluation of different mass features, such as location, signal intensity on T1- and T2-weighted images, contrast enhancement and its pattern and by the presence/ absence of imaging characteristics like mass effect, edema, cysts, necrotic and hemorrhagic areas and dural tail sign (8).

Radiation therapy has been described as an important therapeutic approach in the treatment of intracranial tumours in dogs, resulting in longer survival times in comparison with other treatment modalities $(4,7,9,10)$. Radiation doses and regimens applied to cancer patients depend on tumour histological type and tumour location. Therefore, depending on patient characteristics, owner availability and other constraints, a definitive or a palliative protocol is chosen (11).

The prognosis depends not only on the treatment type, but also on several factors such as the clinical signs at presentation (12), the cerebral involvement (13), the histotype (13-15) and the tumour location (14-16). However, this information is not consensual, which claims for 
additional clinical studies on this topic. Therefore, the major aims of this retrospective study were to characterize, from a clinical point of view, a group of dogs diagnosed with intracranial tumours (meningiomas and gliomas) treated with radiation therapy and to assess the clinical impact of the tumour diagnosis and the radiation protocol elected (definitive $v s$. palliative) on the patient survival.

\section{Patients and Methods}

Inclusion criteria and data processing. The archived medical records were searched for all dogs with intracranial neoplastic disease treated with radiation in VRCC Veterinary Referrals (now rebranded as Southfields Veterinary Specialists) between January 2011 and December 2015. The patients were included only if they had a presumptive diagnosis of meningioma or glioma made by a board-certified veterinary radiologist; completed a radiation therapy course as a primary treatment for their intracranial condition; and had their radiation treatment protocol finished by the end of December 2015. Dogs were excluded if surgery or intravenous chemotherapy were also performed; a full imaging report was lacking or there were doubts in the established diagnosis; if the diagnosis was consistent with another type of primary intracranial tumour, such as choroid plexus tumour, ependymoma, pituitary tumour or any other miscellaneous brain neoplasm; and if the radiotherapy protocol was interrupted or not completed by the end of the study period.

For each dog, patient features were evaluated, such as age, breed, size (small, medium or large), weight, body condition (underweight, ideal weight or overweight), gender (female or male) and reproductive status (intact or neutered). Weight change during treatment period was also analyzed, as done in a recent study (17), distinguishing between cases of critical weight loss ( $\geq 5 \%$ decrease in body weight), stable weight (weight variation $<5 \%$ ) or weight gain ( $\geq 5 \%$ increase in body weight).

Several other variables related to diagnostic procedures were also investigated, such as the reason for consultation (seizures, other neurological signs or seizures and other neurological signs), neurological examination at presentation (normal or abnormal), presumptive diagnosis (meningioma or glioma) and tumour location (supratentorial or infratentorial). The type of radiation protocol used (definitive or palliative) was also studied and the survival time was measured, in days, from the beginning of the treatment (first fraction of radiation therapy) until death for any causes.

Diagnosis and therapeutic approach. Tumour diagnosis was reviewed in all patients through the evaluation of the MRI images and respective reports previously done at the referring veterinary centers. Imaging features such as location, tumour borders, signal intensities, contrast-enhancement, mass effect, bone changes and the presence of cystic regions and dural tail sign were considered for this purpose. These findings were associated with clinical and epidemiological data to confirm and establish an accurate diagnosis.

Regarding treatment, all patients underwent a positioning CT prior to radiation. Computer-assisted plans were all performed by a diplomate in Radiation Oncology. The patients were treated with 3D-conformal external beam megavoltage radiation, which was delivered with a $6 \mathrm{MV}$ linear accelerator (Clinac 600C, Varian). Definitive protocols were organized in 12 to 16 fractions, from
Monday to Friday or every other day (M-W-F) and had a total dose of treatment between 44 and $48 \mathrm{~Gy}$ and a dose per fraction between 2.75 and $3.75 \mathrm{~Gy}$. Palliative protocols were planned in 4 to 8 fractions, in a weekly schedule, with a total treatment dose between 31.5 and 38 Gy and a dose per fraction between 4.75 and 8 Gy.

Follow-up and monitoring. In general, each animal had the first reevaluation at 2-4 weeks after the end of the radiotherapy course, followed by reevaluations every 3 months, on a regular basis until the end of its life. Each evaluation was focused in physical and neurological examination, reassessment of current medication and blood collection to assess phenobarbital levels and liver enzymes. Imaging rechecks were advised to take place firstly at 2-3 months after treatment and then every 6-12 months for tumour monitoring. The clinical follow-up, which also included phone contact with the local veterinarians, was carried out from the beginning of the study until October 22, 2020.

Statistical analysis. The JMP software (15 th version; SAS Institute Inc.) was used for statistical analysis. The results were expressed in absolute and relative frequencies and for the continuous variables, mean, standard deviation and range were also obtained. Comparisons between categorical variables and their association with diagnosis were performed using the Chi-square test and Fisher's exact test, as appropriate. The survival analysis was calculated by the Kaplan-Meier method: survival times were expressed as median values followed by the $95 \%$ confidence interval (CI) and their association with different patient, diagnostic and treatment features was studied by the log-rank test. Only univariate analysis was performed. Associations were considered significant when $p$-values $<0.05$.

\section{Results}

Thirty-seven patients were initially identified. Five were eventually excluded for different reasons: two died during the treatment and did not complete the protocol, two were still receiving treatment at the time of submission and one underwent surgery prior to radiation. Thus, thirty-two dogs $(n=32)$ were included, 16 diagnosed with meningioma and 16 with glioma, whose clinical files were reviewed and the available information recorded.

Patient, diagnostic and treatment features. Mean age was $9.43 \pm 2.69$ years (range $=4-16$ years) to all dogs, $9.00 \pm 3.06$ years (range $=4-16$ years) in the glioma group and $9.88 \pm 2.28$ years (range $=5-14$ years) in the meningioma group. Twentyfive patients $(78.1 \%)$ were purebred and seven $(21.9 \%)$ considered as a crossbred. The most frequent purebreds were Boxer $(n=11)$, Labrador retriever $(n=4)$ and Staffordshire bull terrier $(n=2)$, in addition to the following breeds with only one dog each: Bedlington terrier, Border terrier, Boston terrier, Jack Russel terrier, German shepherd, Samoyed, Shih tzu and Spinone.

Mean weight was $26.84 \pm 10.45 \mathrm{~kg}$ (range $=7-46 \mathrm{~kg}$ ) to all patients, $28.31 \pm 11.88 \mathrm{~kg}$ (range $=7-46 \mathrm{~kg}$ ) in the glioma group and $25.38 \pm 8.94 \mathrm{~kg}$ (range $=8-42 \mathrm{~kg}$ ) in the meningioma 
Table I. Association between patient, diagnostic and therapeutic features with tumour diagnosis (glioma versus meningioma).

\begin{tabular}{|c|c|c|c|c|c|}
\hline & \multicolumn{2}{|c|}{ Glioma } & \multicolumn{2}{|c|}{ Meningioma } & \multirow[t]{2}{*}{$p$-Value } \\
\hline & $\mathrm{n}$ & $\%$ & $\mathrm{n}$ & $\%$ & \\
\hline Age $(n=32)$ & & & & & $p=0.480$ \\
\hline$<10$ years & 9 & $56.25 \%$ & 6 & $37.50 \%$ & \\
\hline$\geq 10$ years & 7 & $43.75 \%$ & 10 & $62.50 \%$ & \\
\hline Breed $(n=32)$ & & & & & $p=0.394$ \\
\hline Purebred & 11 & $68.75 \%$ & 14 & $87.50 \%$ & \\
\hline Crossbred & 5 & $31.25 \%$ & 2 & $12.50 \%$ & \\
\hline Size $(n=32)$ & & & & & $p=0.633$ \\
\hline Small & 3 & $18.75 \%$ & 2 & $12.50 \%$ & \\
\hline Medium & 2 & $12.50 \%$ & 4 & $25.00 \%$ & \\
\hline Large & 11 & $68.75 \%$ & 10 & $62.50 \%$ & \\
\hline Body condition $(n=32)$ & & & & & $p=0.208$ \\
\hline Underweight & 2 & $12.50 \%$ & 1 & $6.25 \%$ & \\
\hline Ideal weight & 6 & $37.50 \%$ & 11 & $68.75 \%$ & \\
\hline Overweight & 8 & $50.00 \%$ & 4 & $25.00 \%$ & \\
\hline Gender $(n=32)$ & & & & & $p=0.073$ \\
\hline Female & 4 & $25.00 \%$ & 10 & $62.50 \%$ & \\
\hline Male & 12 & $75.00 \%$ & 6 & $37.50 \%$ & \\
\hline Reproductive condition $(\mathrm{n}=32)$ & & & & & $p=0.654$ \\
\hline Intact & 4 & $25.00 \%$ & 2 & $12.50 \%$ & \\
\hline Neutered & 12 & $75.00 \%$ & 14 & $87.50 \%$ & \\
\hline Reason for consultation $(n=32)$ & & & & & $p=0.256$ \\
\hline Seizures & 13 & $81.25 \%$ & 9 & $56.25 \%$ & \\
\hline Other neurological signs & 2 & $12.50 \%$ & 6 & $37.50 \%$ & \\
\hline Seizures + other neurological signs & 1 & $6.25 \%$ & 1 & $6.25 \%$ & \\
\hline Neurological examination $(n=26)$ & & & & & $p=0.115$ \\
\hline Normal & 9 & $69.23 \%$ & 4 & $30.77 \%$ & \\
\hline Abnormal & 4 & $30.77 \%$ & 9 & $69.23 \%$ & \\
\hline Tumour location $(n=32)$ & & & & & $p=0.083$ \\
\hline Supratentorial & 15 & $93.75 \%$ & 10 & $62.50 \%$ & \\
\hline Infratentorial & 1 & $6.25 \%$ & 6 & $37.50 \%$ & \\
\hline Radiation therapy protocol $(n=32)$ & & & & & $p=0.704$ \\
\hline Definitive & 10 & $62.50 \%$ & 12 & $75.00 \%$ & \\
\hline Palliative & 6 & $37.50 \%$ & 4 & $25.00 \%$ & \\
\hline Treatment schedule $(n=32)$ & & & & & $p=0.725$ \\
\hline Monday to Friday (M-F) & 9 & $56.25 \%$ & 11 & $68.75 \%$ & \\
\hline Monday, Wednesday and Friday (M-W-F) & 2 & $12.50 \%$ & 1 & $6.25 \%$ & \\
\hline Weekly & 5 & $31.25 \%$ & 4 & $25.00 \%$ & \\
\hline
\end{tabular}

$\mathrm{n}$ : Number of patients; \%: percentage of patients.

group. In most patients $(n=24)$ body weight did not significantly change $(<5 \%)$ between the first and the last radiotherapy fraction. Even so, five dogs lost $5 \%$ or more of their body weight, while other three gained weight on the same proportion. Weight change did not show a statistically significant association with the tumour location $(p=0.610)$, tumour diagnosis $(p=0.705)$, type of treatment protocol chosen $(p=0.840)$ or even survival time $(p=0.891)$.

Table I shows the relative frequencies of other features related to patient signalment, such as size, body condition, gender and reproductive condition. None of the factors showed a statistically significant association with tumour diagnosis.
However, gender showed a value trending towards significance $(p=0.073)$, with a higher representation of males in the group diagnosed with glial tumours (12 out of 16) and females in the group diagnosed with meningeal tumours (10 out of 16$)$.

Regarding the diagnosis, seizures were considered as the main reason for consultation, but other neurological signs were observed, such as ataxia $(n=5)$, head tilt $(n=3)$, ophthalmic/ocular changes $(n=3)$, postural deficits $(n=2)$, nonambulatory tetraparesis $(n=2)$, facial asymmetry $(n=2)$, sudden behavior change $(n=2)$, circling $(n=1)$, depression $(n=1)$ and head pressing $(n=1)$ (Table I). Most of the patients $(62.5 \%)$ were treated with a definitive protocol on a Monday to Friday 
Table II. Kaplan-Meier univariate analysis: Association between patient, diagnostic and therapeutic features and the survival time.

\begin{tabular}{|c|c|c|c|c|c|}
\hline & $\mathrm{n}$ & Mean ST & Median ST & $95 \% \mathrm{CI}$ & $p$-Value \\
\hline Age $(n=30)$ & & & & & $p=0.315$ \\
\hline$<10$ years & 14 & 644.1 & 496 & $249-855$ & \\
\hline$\geq 10$ years & 16 & 487.0 & 524 & $120-588$ & \\
\hline Breed $(n=30)$ & & & & & $p=0.215$ \\
\hline Purebred & 24 & 609.4 & 524 & $287-682$ & \\
\hline Crossbred & 6 & 364.0 & 338.5 & $63-685$ & \\
\hline Size $(n=30)$ & & & & & $p=0.445$ \\
\hline Small & 5 & 453.8 & 346 & $63-904$ & \\
\hline Medium & 4 & 397.0 & 424 & $63-677$ & \\
\hline Large & 21 & 616.8 & 538 & $279-682$ & \\
\hline Body condition $(\mathrm{n}=30)$ & & & & & $p=0.426$ \\
\hline Underweight & 3 & 371.0 & 512 & $63-538$ & \\
\hline Ideal weight & 16 & 545.1 & 499 & $249-682$ & \\
\hline Overweight & 11 & 634.1 & 557 & $101-855$ & \\
\hline Gender $(n=30)$ & & & & & $p=0.117$ \\
\hline Female & 12 & 714.8 & 632.5 & $163-1022$ & \\
\hline Male & 18 & 457.4 & 435 & $246-557$ & \\
\hline Reproductive condition $(\mathrm{n}=30)$ & & & & & $p=0.442$ \\
\hline Intact & 5 & 716.2 & 584 & $249-1357$ & \\
\hline Neutered & 25 & 529.2 & 462 & $279-677$ & \\
\hline Diagnosis $(\mathrm{n}=30)$ & & & & & $p=0.698$ \\
\hline Glioma & 15 & 516.5 & 512 & $101-682$ & \\
\hline Meningioma & 15 & 604.1 & 536 & $249-677$ & \\
\hline Tumour location $(n=30)$ & & & & & $p=0.414$ \\
\hline Supratentorial & 23 & 590.9 & 536 & $279-682$ & \\
\hline Infratentorial & 7 & 460.0 & 462 & $82-677$ & \\
\hline Radiation therapy protocol $(n=30)$ & & & & & $p=0.130$ \\
\hline Definitive & 20 & 639.2 & 560 & $279-754$ & \\
\hline Palliative & 10 & 402.6 & 449 & $63-557$ & \\
\hline
\end{tabular}

n: Number of patients; CI: confidence interval; ST: survival time.

schedule. Radiotherapy dosimetry plans were not available for review. Beam arrangements included 2 to 4 beams with lead block and dynamic wedges used when appropriate. Mean treatment and fraction doses were 43.30 \pm 5.77 Gy (range=31.5$48 \mathrm{~Gy}$ ) and 4.18 \pm 1.85 (range=2.75-8 Gy), respectively.

Follow-up and survival time. For survival analysis, only 30 patients were considered, since one was lost during the clinical follow-up and another one died at home without a specific date. The cause of death was classified either as tumour-related or non-tumour-related. In 18 animals (60\%), death was a result of deterioration of neurological signs, while $7(23 \%)$ died due to other pathological conditions not associated with the intracranial neoplasm, such as cardiovascular conditions $(n=3)$, neoplastic diseases not related with the primary brain tumour $(n=3)$ and debilitating signs from old age $(n=1)$. In 5 dogs $(17 \%)$, the cause of death was not properly defined or was considered unspecific, since clinical investigation was not done.

Median survival time was 524 days (95\% CI=287-677) for all patients, 512 days (95\% CI=101-682) for dogs diagnosed with gliomas and 536 days (95\% CI=249-677) for dogs diagnosed with meningiomas. From our data (Table II), it is possible to verify that neither tumour diagnosis $(p=0.698)$ nor the type of radiation therapy protocol $(p=0.130)$ revealed statistically significant association with survival time (Figures 1 and 2). The 1-, 2- and 3-year survival rates were $63 \%(n=19), 23 \%(n=7)$ and $10.0 \%(n=3)$, respectively.

\section{Discussion}

This study characterized canine patients with presumptive gliomas and meningiomas and showed the survival advantage of radiation therapy, when used as sole treatment. Starting with the diagnosis, the two tumour types showed equal frequencies $(n=16)$, contrary to what we would expect, since meningioma has been reported with a higher relative frequency $(2,4,18)$.

The mean age of all dogs (9.44 years) is in line with similar studies $(4,18,19)$, which is also valid for the age obtained particularly in patients with glioma $(18,20,21)$ and meningioma $(18,21-23)$. This study confirms, once more, 


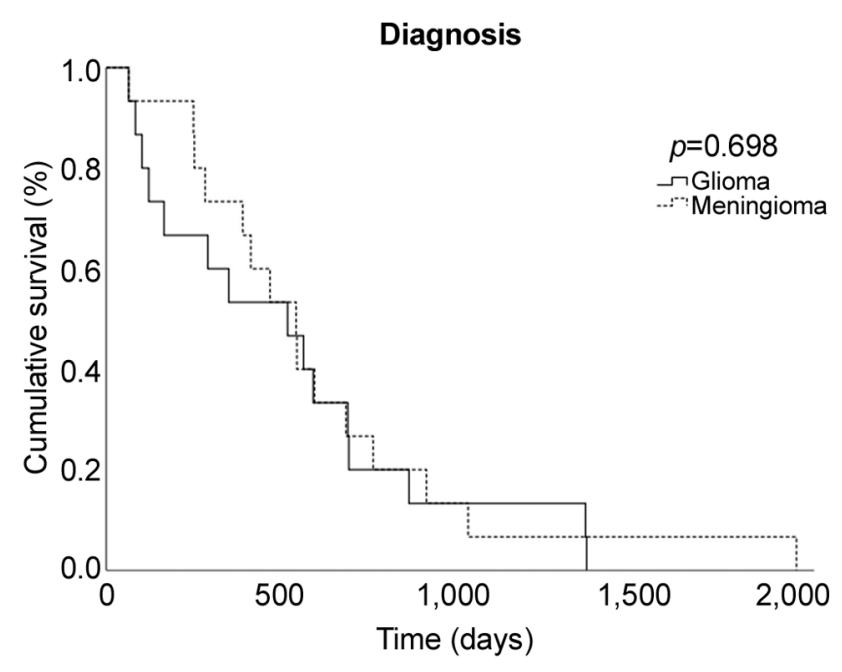

Figure 1. Cumulative overall survival by histological type (glioma versus meningioma).

the tendency of appearance of these tumours in middle-aged to senior dogs, although the same may occur at earlier ages (10 animals were diagnosed under 9 years). Despite the fact that mean age for dogs with meningioma was higher than those who had glioma, its association with the diagnosis did not prove statistical significance, contrary to what was previously noted $(18,24)$.

Boxers represented the most frequent purebred in the study group, which is in agreement with the reported breed predilection of these dogs for intracranial tumours $(4,18$, 21). However, the actual predisposition could not be determined in this study since the proportion of boxers in the general canine population was not addressed. Out of the purebreds, there is a considerable percentage of mixed breed patients, as included in other publications $(16,18,22)$.

In regards of size and body condition, there was a predominance of large breeds and overweight dogs, which resulted in a mean weight of $26.8 \mathrm{~kg}$. Although a slightly higher, this result is very similar to most previous findings $(3,4,19)$. Even so, a recent study carried out in Japan showed a higher prevalence of primary brain tumours in small to medium-sized breeds (21). This opposed result must be interpreted considering the differences between Asian and European countries regarding to their canine population.

Only a small percentage of patients showed a critical weight loss during treatment period, which is in accordance with another study recently published (17). Although weight change did not show a significant association with the type of protocol, four out of five patients who had a critical weight loss were hospitalized on a Monday to Friday schedule for their definitive treatment protocol. These conditions may have led to greater stress and reluctance to

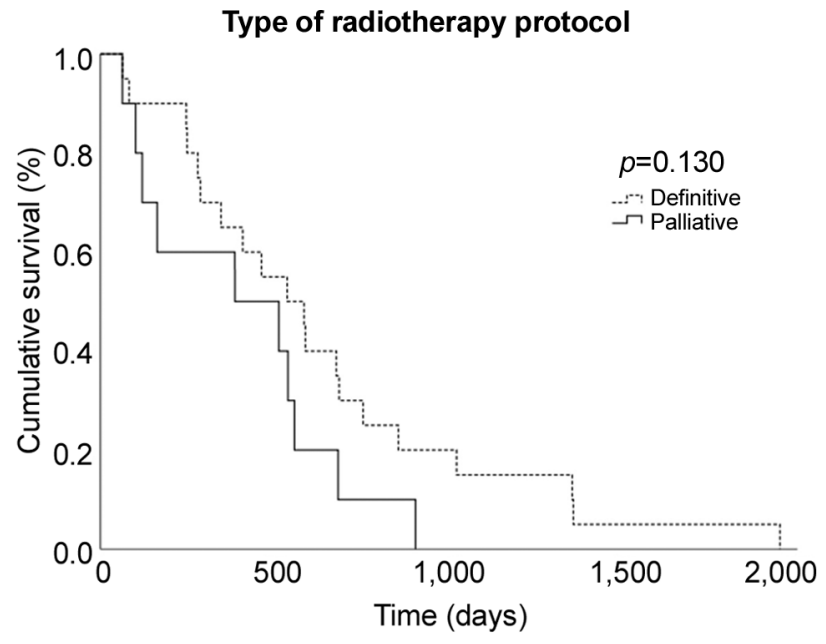

Figure 2. Cumulative overall survival by type of radiotherapy protocol (definitive versus palliative).

eat, which could explain this loss. Howsoever, it should be noted that the majority $(n=24)$ maintained their weight and three dogs had a weight gain greater than 5\%, which demonstrates the negligible impact of the therapy on the animal's quality of life, especially with regard to appetite and ability to eat.

No features related to the patient, the diagnosis or even the selected treatment protocol, showed a significant association with the type of tumour diagnosed. However, gender exhibited a value trending to significance (females with higher probability to have meningiomas, but without reaching the statistical significance; $p=0.073$ ), which raises the question that if we had included more dogs, as well as an equivalent number of individuals of both genders, perhaps a significant result could have been achieved. If so, we could have reinforced the suspicion that a breed predilection to canine brain tumours maybe exists, as has already been proven in human medicine, with a greater predisposition of women to meningiomas (25).

With respect to survival time, the overall median value of 524 days is superior compared with the previous value of 351 days reached by $\mathrm{Hu}$ et al. (15) after their review of 13 trials, in which radiation therapy was elected. Considering a more current review article, our results are in line with those achieved more recently for this type of treatment and far superior to the outcome reached by other treatment options, such as surgery and chemotherapy (1).

Meningiomas are associated in the literature with a significantly better prognosis, in terms of survival, compared to gliomas $(13,26)$. Despite this idea, in our study, both groups showed a good outcome: 512 and 536 days for presumptive gliomas and meningiomas, respectively. These 
results showed the benefit of radiation not only in the treatment of meningiomas, but also in the treatment of gliomas, which may suggest a change in the way we should approach the intra-axial and suspected glial tumours and establish their prognosis. We also hypothesized a possible superior benefit of the definitive protocol over the palliative in terms of survival, however our results did not show a statistically significant difference.

Various limitations have been assigned to this type of studies, such as small case number, lack of a definitive histological diagnosis and other concerns related to the retrospective nature of the study, such as incomplete reporting on radiation therapy planning. In fact, despite our number of cases being comparable to the one used by other authors (2, $9,10)$, it may not have been large enough to draw further conclusions with greater statistical and/ or clinical significance. The lack of a definitive diagnosis is other common limitation since the majority of results published in this scope are based on imaging diagnoses, as already mentioned. In our opinion, this is due to the potential risks associated with an ante-mortem biopsy that owners frequently decline, as described in previous works where this procedure has been proposed (6). Even so, with the new, safer and more effective techniques currently being developed (27), we believe that in the next few years, studies with this nature will be more easily based on histological diagnosis. Finally, despite being our purpose to report radiation therapy planning according to Keyerleber et al. (28), this was not possible due to the retrospective nature of the study, which led us to incomplete information in some patients, although the fundamental information (treatment doses, number of fractions, type of schedule and number of fields) has been ensured for all cases. Future studies with a prospective design should be performed to overcome this limitation.

Our study has also some strengths that should be highlighted. Firstly, it allows a complete characterization of a set of patients diagnosed with the two most common types of brain tumours in canine species. Secondly, it contributes with relevant and new information about the efficacy of conventional radiotherapy at a time when most recent literature has focused on more advanced and high technological radiation modalities. And finally, to the best of our knowledge, it is the first study that showed a comparable prognosis for patients with meningiomas and gliomas treated with radiation therapy in the same veterinary referral hospital.

\section{Conclusion}

This study reinforces the idea about the efficacy of radiation therapy in the treatment of both canine glioma and meningioma. Survival times obtained for both tumour types are consistent with other published studies, which are higher than those achieved by other therapeutic approaches.
Furthermore, survival results for the glioma group demonstrate that we should consider more frequently and with more confidence the use of radiation as the primary treatment for these tumours, as has been already done for patients diagnosed with meningiomas.

\section{Conflicts of Interest}

The Authors declare no conflicts of interest with regard to this study.

\section{Authors' Contributions}

Magalhães TR, North S and Queiroga FL conceived and designed the study; Magalhães TR, Benoît J and Néčová S acquired the data; Magalhães TR and Queiroga FL analyzed the data; Magalhães TR wrote the paper; Magalhães TR, Benoît J, Néčová S, North S and Queiroga FL revised the manuscript.

\section{Acknowledgements}

This work received financial support of project UIDB/00211/2020 funded by Fundação para a Ciência e Tecnologia (FCT) through national funds. Authors would also like to thank the support received by project UIDB/04033/2020 from FCT.

The authors would like to acknowledge the Southfields Veterinary Specialists (formerly VRCC Veterinary Referrals) for all the data that make up the basis of this work and also all the clinicians who have referred their cases to be evaluated in this institution.

\section{References}

1 Miller AD, Miller CR and Rossmeis1 JH: Canine primary intracranial cancer: A clinicopathologic and comparative review of glioma, meningioma, and choroid plexus tumors. Front Oncol 9: 1151, 2019. PMID: 31788444. DOI: 10.3389/fonc.2019.01151

2 Spugnini EP, Thrall DE, Price GS, Sharp NJ, Munana K and Page RL: Primary irradiation of canine intracranial masses. Vet Radiol Ultrasound 41(4): 377-380, 2000. PMID: 10955504. DOI: $10.1111 / \mathrm{j} .1740-8261.2000 . t b 02091 . x$

3 Bley CR, Sumova A, Roos M and Kaser-Hotz B: Irradiation of brain tumors in dogs with neurologic disease. J Vet Intern Med 19(6): 849-854, 2005. PMID: 16355679. DOI: 10.1892/08916640(2005)19[849:iobtid]2.0.co;2

4 Treggiari E, Maddox TW, Gonçalves R, Benoit J, Buchholz J and Blackwood L: Retrospective comparison of three-dimensional conformal radiation therapy $v s$. prednisolone alone in 30 cases of canine infratentorial brain tumors. Vet Radiol Ultrasound 58(1): 106-116, 2017. PMID: 27859957. DOI: 10.1111/vru.12440

5 Brearley MJ, Jeffery ND, Phillips SM and Dennis R: Hypofractionated radiation therapy of brain masses in dogs: A retrospective analysis of survival of 83 cases (1991-1996). J Vet Intern Med 13(5): 408-412, 1999. PMID: 10499721. DOI: 10.1892/0891-6640(1999)013<0408:hrtobm>2.3.co;2

6 Griffin LR, Nolan MW, Selmic LE, Randall E, Custis J and LaRue S: Stereotactic radiation therapy for treatment of canine intracranial meningiomas. Vet Comp Oncol 14(4): e158-e170, 2016. PMID: 25524449. DOI: $10.1111 /$ vco.12129 
7 Monforte Monteiro SR, Rossmeisl JH, Russell J, Holmes MA, Wessmann A, Morris J, Dobson JM and Vanhaesebrouck AE: Effect of radiotherapy on freedom from seizures in dogs with brain tumors. J Vet Intern Med 34(2): 821-827, 2020. PMID: 32032456. DOI: 10.1111/jvim.15695

8 Ródenas S, Pumarola M, Gaitero L, Zamora A and Añor S: Magnetic resonance imaging findings in $40 \mathrm{dogs}$ with histologically confirmed intracranial tumours. Vet J 187(1): 8591, 2011. PMID: 19914851. DOI: 10.1016/j.tvj1.2009.10.011

9 Axlund TW, McGlasson ML and Smith AN: Surgery alone or in combination with radiation therapy for treatment of intracranial meningiomas in dogs: 31 cases (1989-2002). J Am Vet Med Assoc 221(11): 1597-1600, 2002. PMID: 12479332. DOI: 10.2460/javma.2002.221.1597

10 Keyerleber MA, McEntee MC, Farrelly J, Thompson MS, Scrivani PV and Dewey CW: Three-dimensional conformal radiation therapy alone or in combination with surgery for treatment of canine intracranial meningiomas. Vet Comp Oncol 13(4): 385-397, 2015. PMID: 23869984. DOI: 10.1111/vco.12054

11 McEntee MC: Veterinary radiation therapy: Review and current state of the art. J Am Anim Hosp Assoc 42(2): 94-109, 2006. PMID: 16527910 . DOI: 10.5326/0420094

12 Jeffery $\mathrm{N}$ and Brearley MJ: Brain tumours in the dog: Treatment of 10 cases and review of recent literature. Journal of Small Animal Practice 34(8): 367-372, 1993. DOI: 10.1111/j.17485827.1993.tb02720.x

13 Heidner GL, Kornegay JN, Page RL, Dodge RK and Thrall DE: Analysis of survival in a retrospective study of 86 dogs with brain tumors. J Vet Intern Med 5(4): 219-226, 1991. PMID: 1941756. DOI: 10.1111/j.1939-1676.1991.tb00952.x

14 Klopp LS and Rao S: Endoscopic-assisted intracranial tumor removal in dogs and cats: Long-term outcome of 39 cases. J Vet Intern Med 23(1): 108-115, 2009. PMID: 19175729. DOI: 10.1111/j.1939-1676.2008.0234.x

$15 \mathrm{Hu} \mathrm{H}$, Barker A, Harcourt-Brown T and Jeffery N: Systematic review of brain tumor treatment in dogs. J Vet Intern Med 29(6): 1456-1463, 2015. PMID: 26375164. DOI: 10.1111/jvim.13617

16 Rossmeisl JH Jr., Jones JC, Zimmerman KL and Robertson JL: Survival time following hospital discharge in dogs with palliatively treated primary brain tumors. J Am Vet Med Assoc 242(2): 193-198, 2013. PMID: 23276095. DOI: 10.2460/ javma.242.2.193

17 Callanan GF, Pfeiffer I and Smith K: Evaluation of weight loss in canine cancer bearing patients undergoing radiation therapy. Vet Comp Oncol 18(2): 184-190, 2020. PMID: 31419017. DOI: $10.1111 /$ vco. 12528

18 Snyder JM, Shofer FS, Van Winkle TJ and Massicotte C: Canine intracranial primary neoplasia: 173 cases (1986-2003). J Vet Intern Med 20(3): 669-675, 2006. PMID: 16734106. DOI: 10.1892/0891-6640(2006)20[669:cipnc]2.0.co;2

19 Bley CR, Meier V, Schwarz P, Roos M and Besserer J: A complication probability planning study to predict the safety of a new protocol for intracranial tumour radiotherapy in dogs. Vet Comp Oncol 15(4): 1295-1308, 2017. PMID: 27576304. DOI: $10.1111 /$ vco. 12265
20 Young BD, Levine JM, Porter BF, Chen-Allen AV, Rossmeisl JH, Platt SR, Kent M, Fosgate GT and Schatzberg SJ: Magnetic resonance imaging features of intracranial astrocytomas and oligodendrogliomas in dogs. Vet Radiol Ultrasound 52(2): 132-141, 2011. PMID: 21388463. DOI: 10.1111/j.1740-8261.2010.01758.x

21 Kishimoto TE, Uchida K, Chambers JK, Kok MK, Son NV, Shiga T, Hirabayashi $\mathrm{M}$, Ushio $\mathrm{N}$ and Nakayama $\mathrm{H}$ : A retrospective survey on canine intracranial tumors between 2007 and 2017. J Vet Med Sci 82(1): 77-83, 2020. PMID: 31801930. DOI: $10.1292 /$ jvms.19-0486

22 Montoliu P, Añor S, Vidal E and Pumarola M: Histological and immunohistochemical study of 30 cases of canine meningioma. J Comp Pathol 135(4): 200-207, 2006. PMID: 17049358. DOI: 10.1016/j.jcpa.2006.06.006

23 Sturges BK, Dickinson PJ, Bollen AW, Koblik PD, Kass PH, Kortz GD, Vernau KM, Knipe MF, Lecouteur RA and Higgins RJ: Magnetic resonance imaging and histological classification of intracranial meningiomas in 112 dogs. J Vet Intern Med 22(3): 586-595, 2008. PMID: 18466258. DOI: 10.1111/j.19391676.2008.00042.x

24 Schwartz M, Lamb CR, Brodbelt DC and Volk HA: Canine intracranial neoplasia: Clinical risk factors for development of epileptic seizures. J Small Anim Pract 52(12): 632-637, 2011. PMID: 21954970. DOI: 10.1111/j.1748-5827.2011.01131.x

25 Sun T, Plutynski A, Ward S and Rubin JB: An integrative view on sex differences in brain tumors. Cell Mol Life Sci 72(17): 3323-3342, 2015. PMID: 25985759. DOI: 10.1007/s00018-015$1930-2$

26 Schwarz P, Meier V, Soukup A, Drees R, Besserer J, Beckmann $\mathrm{K}$, Roos M and Bley CR: Comparative evaluation of a novel, moderately hypofractionated radiation protocol in $56 \mathrm{dogs}$ with symptomatic intracranial neoplasia. J Vet Intern Med 32(6): 2013-2020, 2018. PMID: 30308086. DOI: 10.1111/jvim.15324

27 Kani Y, Cecere TE, Lahmers K, LeRoith T, Zimmerman KL, Isom S, Hsu FC, Debinksi W, Robertson JL and Rossmeisl JH: Diagnostic accuracy of stereotactic brain biopsy for intracranial neoplasia in dogs: Comparison of biopsy, surgical resection, and necropsy specimens. J Vet Intern Med 33(3): 1384-1391, 2019. PMID: 30990928. DOI: 10.1111/jvim.15500

28 Keyerleber MA, McEntee MC, Farrelly J and Podgorsak M: Completeness of reporting of radiation therapy planning, dose, and delivery in veterinary radiation oncology manuscripts from 2005 to 2010. Vet Radiol Ultrasound 53(2): 221-230, 2012. PMID: 22092592. DOI: 10.1111/j.1740-8261.2011.01882.x

Received November 17, 2020

Revised November 29, 2020 Accepted December 4, 2020 